\title{
Lipid peroxidation and oxidant stress regulate hepatic apolipoprotein B degradation and VLDL production
}

\author{
Meihui Pan, ${ }^{1,2}$ Arthur I. Cederbaum, ${ }^{3}$ Yuan-Li Zhang, ${ }^{4}$ Henry N. Ginsberg, ${ }^{4}$ Kevin Jon Williams, ${ }^{5}$
} and Edward A. Fisher1,2

\begin{abstract}
${ }^{1}$ Laboratory of Lipoprotein Research, The Zena and Michael A. Wiener Cardiovascular Institute and the Department of Medicine, Mount Sinai School of Medicine, New York, New York, USA. "Marc and Ruti Bell Vascular Biology and Disease Program, Leon H. Charney Division of Cardiology, Department of Medicine; and Department of Cell Biology; New York University School of Medicine, New York, New York, USA. ${ }^{3}$ Department of Pharmacology and Biological Chemistry, Mount Sinai School of Medicine, New York, New York, USA. ${ }^{4}$ Department of Medicine, College of Physicians and Surgeons, Columbia University, New York, New York, USA. 5Dorrance H. Hamilton Research Laboratories, Division of Endocrinology, Diabetes and Metabolic Diseases, Department of Medicine, Jefferson Medical College, Thomas Jefferson University, Philadelphia, Pennsylvania, USA.
\end{abstract}

\begin{abstract}
How $\omega-3$ and $\omega-6$ polyunsaturated fatty acids (PUFAs) lower plasma lipid levels is incompletely understood. We previously showed that marine $\omega-3$ PUFAs (docosahexaenoic acid [DHA] and eicosapentaenoic acid) stimulate a novel pathway, post-ER presecretory proteolysis (PERPP), that degrades apolipoprotein B100 (ApoB100), thereby reducing lipoprotein secretion from liver cells. To identify signals stimulating PERPP, we examined known actions of $\omega-3$ PUFA. In rat hepatoma or primary rodent hepatocytes incubated with $\omega-3$ PUFA, cotreatment with the iron chelator desferrioxamine, an inhibitor of iron-dependent lipid peroxidation, or vitamin E, a lipid antioxidant, suppressed increases in thiobarbituric acid-reactive substances (TBARSs; a measure of lipid peroxidation products) and restored ApoB100 recovery and VLDL secretion. Moreover, $\omega-6$ and nonmarine $\omega-3$ PUFA, also prone to peroxidation, increased ApoB100 degradation via intracellular induction of TBARSs. Even without added fatty acids, degradation of ApoB100 in primary hepatocytes was blocked by desferrioxamine or antioxidant cotreatment. To extend these results in vivo, mice were infused with DHA, which increased hepatic TBARSs and reduced VLDL-ApoB100 secretion. These results establish a novel link between lipid peroxidation and oxidant stress with APoB100 degradation via PERPP, and may be relevant to the hypolipidemic actions of dietary PUFAs, the basal regulation of ApoB100 secretion, and hyperlipidemias arising from ApoB100 overproduction.
\end{abstract}

\section{Introduction}

Apolipoprotein B100 (ApoB100) is the critical protein component of VLDL, LDL, and lipoprotein (a), the major atherogenic lipoproteins derived from human liver. Assembly of ApoB100 into lipoproteins begins when the nascent polypeptide translocates into the ER lumen, where it associates with lipids in a process facilitated by the microsomal triglyceride transfer protein (MTP). Unlike synthesis of the majority of hepatic secretory proteins, that of ApoB100 is nearly constitutive, and its secretion is regulated primarily by co- and posttranslational degradation (for a recent review, see ref. 1).

We recently characterized a novel pathway for the degradation of newly synthesized ApoB100 within cells of hepatic origin (2). This pathway, which we have called post-ER presecretory proteolysis (PERPP), acts after the early MTP-dependent steps of lipoprotein assembly and preferentially targets large (lipid-rich) ApoB-lipoproteins and decreases their secretion. Importantly, PERPP is dis-

Nonstandard abbreviations used: apolipoprotein B100 (ApoB100); apolipoprotein $\mathrm{E}$ (ApoE); arachidonic acid (AA); butylated hydroxytoluene (BHT); desferrioxamine (DFX); diethylenetriaminepentaacetic acid (DTPA); docosahexaenoic acid (DHA); eicosapentaenoic acid (EPA); ER-associated degradation (ERAD); fatty acid complexed to BSA (FA/BSA); linolenic acid (LNA); malondialdehyde (MDA); McArdle RH7777 (McA); methionine/cysteine (Met/Cys); microsomal triglyceride transfer protein (MTP); myristic acid (MA); oleic acid (OA); polyunsaturated fatty acid (PUFA); postER presecretory proteolysis (PERPP); thiobarbituric acid-reactive substance (TBARS). Conflict of interest: The authors have declared that no conflict of interest exists.

Citation for this article: J. Clin. Invest. 113:1277-1287 (2004).

doi:10.1172/JCI200419197. tinct from the two previously described pathways for degradation of newly synthesized ApoB100, namely, ER-associated degradation (ERAD) and reuptake (1). The PERPP pathway, therefore, fills an important gap in our understanding of the physiologic control of ApoB100 secretion.

The ERAD pathway appears to be most active during cellular deficiency of lipids or of lipid transfer and has been demonstrated in vitro and in vivo $(2,3)$. Under these circumstances, less newly synthesized ApoB100 is required for lipoprotein assembly, and the relative excess is degraded by the proteasome. On the other hand, under conditions where the supply of lipids is not a limitation, ApoB100 secretion can still be regulated at a posttranscriptional level, as exemplified by the changes in lipoprotein secretion when different types of fatty acid are supplied to liver cells (4). Consistent with their dietary effects on plasma lipoprotein levels $(5,6)$, ApoB100 secretion is reduced by the fish oil (marine) $\omega-3$ fatty acids and is increased by saturated fatty acids, with alterations in PERPP being responsible for these changes $(2,4)$. In addition, based on either subcellular fractionation or proteasome inhibitor studies, PERPP may also be responsible for alterations in ApoB100 secretion caused by other metabolic factors, such as the administration of insulin (7), insulin resistance (8), and choline deficiency (9).

The goal of the present study was to discover the biochemical basis for the stimulation of PERPP by marine $\omega-3$ fatty acids. Six actions of these fatty acids have been reported in the literature, and each action was considered whether or not it was already known to alter VLDL secretion. Two of these, suppression of tri- 
glyceride synthesis $(10,11)$ and stimulation of PPAR $\alpha(12)$, were not a focus here, because there is already evidence against their participation in effects of $\omega-3$ fatty acids on hepatic ApoB100 output (e.g., refs. 13, 14). Thus, we examined the other four actions: stimulation of LDL receptor activity $(15,16)$; action as precursors of eicosanoids (17); stimulation of $\mathrm{Ca}^{2+}$ flux $(18,19)$; and, because of the presence of multiple unsaturated bonds, action as robust substrates for lipid peroxidation and inducers of oxidant stress. Of these, only LDL receptor activity $(16,20-22)$ and calcium flux (23-25) had been reported to affect the secretion VLDL or ApoB100, although, regarding the LDL receptor, there has been some controversy (e.g., refs. 26, 27).

Surprisingly, the results of the present experiments in vitro and in vivo provide strong evidence that stimulation of ApoB100 degradation by marine $\omega-3$ fatty acids requires increased generation of lipid peroxidation products. Moreover, other easily peroxidized lipid molecules $-\omega-6$ and nonmarine $\omega-3$ polyunsaturated fatty acids (PUFAs) - act similarly. Besides introducing a novel regulatory mechanism for hepatic ApoB-lipoprotein production, the results have significant implications for the mechanisms of the well-known lipid-lowering effect of dietary PUFA, and, as will be explained, for basal regulation of ApoB100 secretion, hyperlipidemias arising from ApoB100 overproduction, and the ineffectiveness of antioxidants in recent clinical trials (28-30).

\section{Results}

The LDL receptor does not contribute to docosabexaenoic acid-induced ApoB100 degradation. In our previous study, we ruled out a role for LDL receptor-mediated cell-surface reuptake (20) in the effects of $\omega-3$ fatty acids on VLDL secretion (2). We therefore sought to investigate a possible role for LDL receptors in any cellular compartment. Primary hepatocytes were prepared from LDL receptor-deficient ( $L d l r$-KO; ref. 31$)$ and wild-type mice. The cells were pretreated with BSA (0.12 mM; control) or docosahexaenoic acid (DHA)/BSA $(0.6 \mathrm{mM} / 0.12 \mathrm{mM})$ for 2 hours. They were then pulse-labeled with $\left[{ }^{35} \mathrm{~S}\right]$-methionine/cysteine ( $\left[{ }^{35} \mathrm{~S}\right]$-Met/Cys) for 30 minutes, and chased (in the presence of BSA or DHA/BSA) for 30 or 120 minutes. As shown in Figure 1A, DHA stimulated substantial degradation of newly synthesized ApoB100 even in the complete absence of cellular LDL receptors. Furthermore, when incubated in the same medium, $L d l r$-KO and wild-type hepatocytes degraded essentially identical amounts of newly synthesized ApoB100 (compare Figure $1 \mathrm{~A}$ with Figure $1 \mathrm{~B}$ ), indicating no detectable role for $\mathrm{LDL}$ receptors under the conditions of this experiment. This may be related to the recent finding that the LDL receptor influences the secretion of small, dense ApoB-lipoproteins (32), whereas PERPP predominantly affects the secretion of larger, buoyant ones (2).

Enzymatic conversion of DHA to eicosanoids is not required for the stimulation of ApoB100 degradation. DHA is a substrate for cyclooxygenase and can give rise to eicosanoids with pleiotropic biological effects (17). In four separate pulse-chase studies, we found that high concentrations of aspirin, a potent cyclooxygenase inhibitor (33), did not impair the ability of DHA to induce ApoB100 degradation (e.g., $30 \% \pm 5 \%$ recovery without aspirin; $33 \% \pm 3 \%$ with $5 \mathrm{mM}$ aspirin). These results are consistent with prior studies indicating that fish oils are relatively poor cyclooxygenase substrates and that cyclooxygenase activity is naturally low in hepatic cells (17).

DHA-induced ApoB100 degradation is independent of calcium flux. DHA can increase intracellular $\mathrm{Ca}^{2+}$ in a number of extrahepatic cell types (e.g., refs. 18, 19), and changes in calcium metabolism have
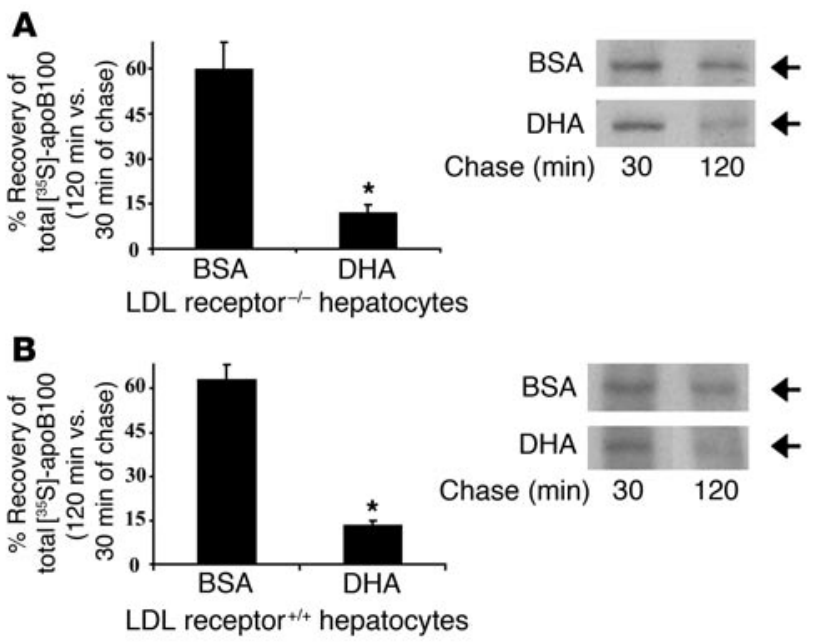

\section{Figure 1}

DHA stimulates ApoB100 degradation in primary hepatocytes independent of the LDL receptor. Primary hepatocytes from Ldlr-KO (A) or wild-type (B) C57BL/6J male mice were maintained overnight in modified Waymouth's medium. Cells were then incubated with either BSA or DHA/BSA complexes and subjected to the pulse-chase protocol described in the text. Chase medium and cell lysate samples were harvested at the indicated time points for immunoprecipitation/SDSPAGE analysis of newly synthesized ApoB100. The fluorograms shown here on the right, as well as in the following figures, were representative of three independent experiments, in each of which at least duplicate wells were analyzed. The graph summarizes the recovery data of total (cell lysate + medium) labeled ApoB100 as determined by densitometric analysis of the fluorograms. The arrows in this and the other figures indicate the position of the ApoB100 band. ${ }^{\star} P<0.001$ vs. BSA.

been reported to affect VLDL production (e.g., refs. 23-25). Therefore, we used several strategies to investigate the role of increased intracellular calcium in the effects of marine $\omega-3$ fatty acids on the degradation of newly synthesized ApoB100 in hepatocytes.

First, to eliminate calcium influx, the calcium-containing medium routinely used was replaced by calcium-free medium (no. 21068-028; Life Technologies Inc., Carlsbad, California, USA) for the entire experiment (pretreatment, pulse, and chase periods). In addition, calcium-free PBS was used to wash the cells. In pulsechase studies in McArdle RH7777 (McA) cells, the recovery of total $\left[{ }^{35} \mathrm{~S}\right]$-ApoB100 was not affected by the absence of calcium from the medium (data not shown). Second, several agents that manipulate intracellular calcium concentrations or flux also had no effect on DHA-stimulated ApoB100 degradation (data not shown): (a) $\mathrm{GdCl}_{3}$ (100 mM), a general blocker of stretch-activated calcium channels (34); (b) thapsigargin $(2 \mu \mathrm{M})$, which depletes the ER calcium pool and increases cytosolic calcium (35); and (c) the calcium ionophore A23187 $(0.5 \mu \mathrm{g} / \mathrm{ml})$, which increases calcium influx (36). Finally, the inhibitor E64d $(10 \mu \mathrm{M})$, which inhibits calpains and other cysteine proteases, had no significant effect on recovery of ApoB100 from DHA-treated McA cells ( $29 \% \pm 6 \%$ without vs. $34 \% \pm 4 \%$ with E64d).

Overall, then, there is no evidence to support DHA-induced perturbations of the influx or intracellular concentration of calcium as the basis for the stimulation of degradation of newly synthesized ApoB100 in hepatocytes.

DHA-stimulated degradation of newly synthesized ApoB100 requires increases in the cellular content of lipid peroxides. Owing to their 

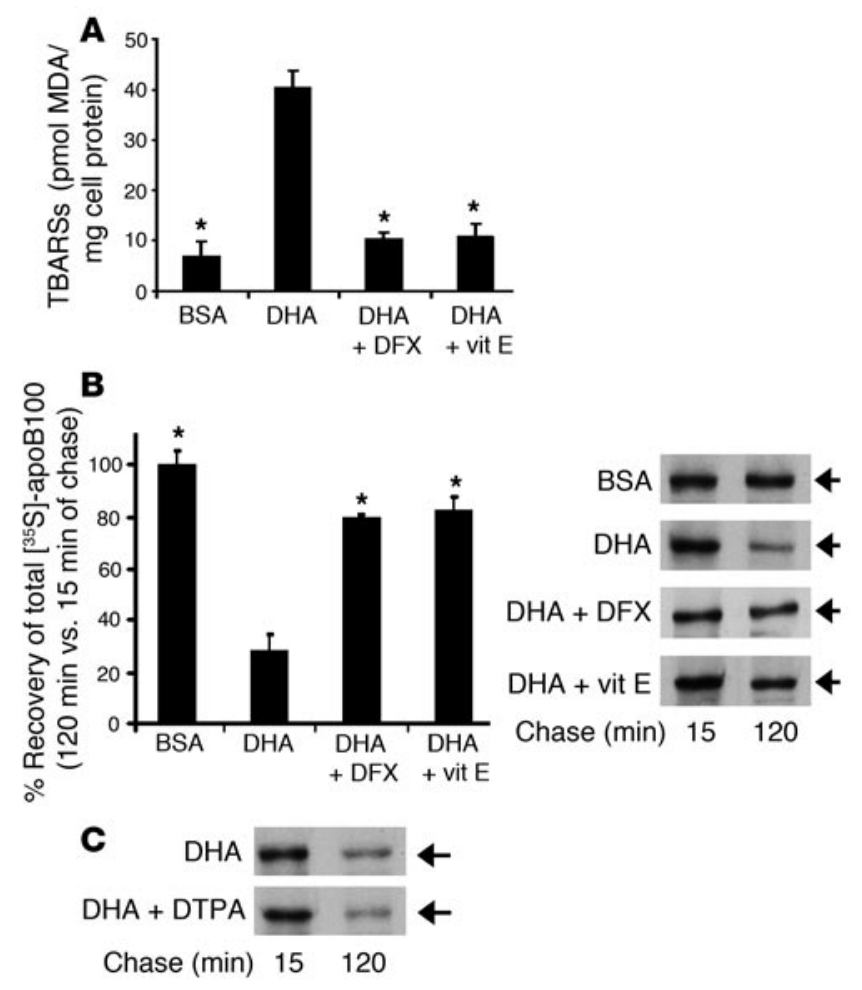

numerous double bonds, eicosapentaenoic acid (EPA) and DHA are excellent substrates for iron-dependent peroxidation reactions that can give rise to lipid peroxides, reactive lipid oxygen species, and increased oxidant stress $(37,38)$. To explore the involvement of lipid peroxidation in DHA-stimulated degradation of newly synthesized ApoB100, we used desferrioxamine (DFX), a specific chelator for iron (39), which inhibits irondependent lipid peroxidation. As shown in Figure 2A, McA cells treated with DHA exhibited a large increase in their content of thiobarbituric acid-reactive substances (TBARSs), a common measure of lipid peroxidation products ( $\sim 4.6$-fold over cells treated with either BSA or oleic acid (OA)/BSA complexes; data not shown), which was completely abolished by cotreatment with DFX. Most importantly, DFX cotreatment also increased the total recovery of $\left[{ }^{35} \mathrm{~S}\right]$-ApoB100 from DHA-treated cells almost threefold relative to the peak incorporation of isotope in the BSA group (Figure 2B). This represented a $72 \%$ inhibition of DHAinduced ApoB100 degradation.

Because DFX passes across the plasma membrane, we next sought to determine whether extracellular iron, intracellular iron, or both are important for the effects of DHA on ApoB100 degradation. Thus, we used a nonpermeable chelator for iron, diethylenetriaminepentaacetic acid (DTPA) (40). Unlike DFX, DTPA failed to inhibit the induction of cellular ApoB100 degradation by DHA (Figure 2C). Consistent with this failure was the inability of DTPA to inhibit the DHA-stimulated increase in TBARSs (data not shown). Therefore, only the cell-permeable chelator DFX protects ApoB100, indicating that the generation of lipid peroxides essential for this process occurs intracellularly.

To verify the key role of lipid peroxidation in ApoB100 degradation, we inhibited this process by a method distinct from iron chelation. Cotreatment of McA cells with the natural lipid antioxidant vitamin E efficiently blocked both the DHA-induced accu-

\section{Figure 2}

Cotreatment of McA cells with DHA and either an intracellular iron chelator or an antioxidant inhibits ApoB100 degradation and the increase in intracellular lipid peroxidation. (A) McA cells were pretreated for 1 hour with medium containing BSA or DHA/BSA complexes, followed by a 3-hour incubation in medium containing BSA, DHA/BSA, DHA/BSA plus $100 \mu \mathrm{M}$ DFX, or DHA/BSA plus $120 \mu \mathrm{M}$ vitamin $\mathrm{E}$ (vit E). Cells were then harvested for TBARS assays. ${ }^{*} P<0.01$ vs. DHA. (B) McA cells were pretreated with BSA or DHA/BSA for 2 hours and then subjected to a pulse-chase study similar to that illustrated in Figure 1 , except that DFX or vitamin E was administered 45 minutes before labeling and was present in the medium thereafter. The composite fluorogram displays representative total labeled ApoB100 recovery data, and the graph summarizes three complete experiments with each treatment applied to triplicate wells. ${ }^{*} P<0.01$ vs. DHA. (C) A similar pulse-chase study was carried out, but DFX was replaced by the cellimpermeable iron chelator DTPA $(100 \mu \mathrm{M})$. Recovery and fluorography of labeled ApoB100 were done as in Figure 1.

mulation of TBARSs in the cells (Figure 2A) and the enhanced degradation of newly synthesized ApoB100 (Figure 2B).

We were interested in whether the effects of DHA on ApoB100 degradation were limited to the treatment time we typically study (4 hours or less). To approach this issue, a longer-term study was undertaken in which McA cells were incubated for up to 12 hours with fatty acid/BSA (FA/BSA) complexes (either DHA/BSA or OA/BSA; because of the prolonged exposure of the cells to unsaturated fatty acid, the final concentration for either was reduced to $0.3 \mathrm{mM}$ to prevent cytotoxicity). After every 4 hours, up to 12 hours, the conditioned medium was collected and fresh medium applied, so that the recovery of labeled ApoB100 from the conditioned medium of the first and last 4-hour intervals could be compared. At the end of the first 4-hour interval, relative to recovery from OA-treated cells, the recovery of labeled ApoB100 from the DHA-treated cells was $49 \% \pm 6 \%$. In the last 4 -hour period, the decrease in ApoB100 recovery from the DHA-treated cells was not only maintained but magnified, to only $15 \% \pm 5 \%$ of the OA-treated value.

Taken together, our data in vitro show that DHA-induced ApoB100 degradation is strongly associated with, and depends on, the substantial cellular accumulation of lipid peroxidation products, and that the effects of DHA on ApoB100 degradation can be sustained for at least 12 hours.

Blockage of DHA-induced lipid peroxidation restores the secretion of ApoB100 on VLDL particles. In our studies of ERAD of ApoB100, we noted that proteasomal inhibitors did not increase the secretion of ApoB-lipoproteins; instead, the rescued ApoB100 remained intracellular, associated with the ER $(41,42)$. Because ApoB100 degradation induced by marine $\omega-3$ fatty acids is mediated by PERPP, a process independent of ERAD (2), we now sought to determine whether the rescue of ApoB100 by DFX or vitamin E also increases the secretion of ApoB100.

For this purpose, we preincubated the McA cells for 1.5 hours with DHA, then labeled the cells to steady state with $\left[{ }^{35} \mathrm{~S}\right]-\mathrm{Met} / \mathrm{Cys}$ for 3 hours. The medium was collected to recover $\left[{ }^{35} \mathrm{~S}\right]-\mathrm{ApoB} 100$. The accumulation of labeled ApoB100 in medium from DHAtreated cells was only $16 \%$ of the BSA control value (Figure $3 \mathrm{~A}$ ), which is consistent with the pulse-chase data (Figure 2). Cotreatment with either DFX or vitamin E substantially increased the secretion of ApoB100 approximately fourfold over that obtained by DHA alone (Figure 3A). 
A
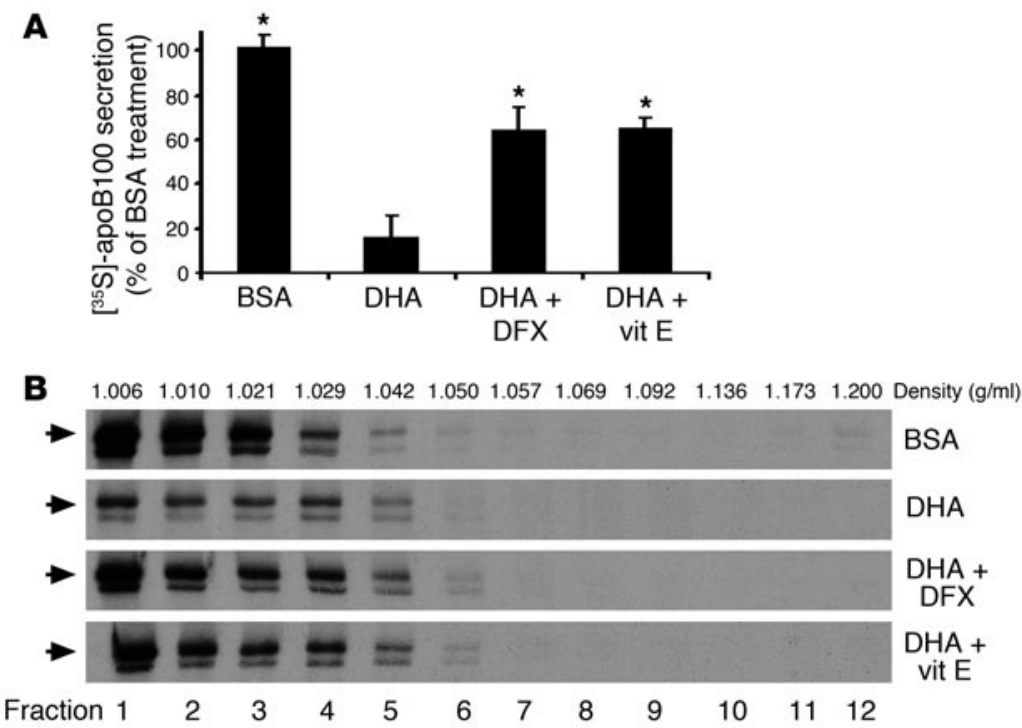

Figure 3

DFX or vitamin E restores the secretion of VLDL from McA cells incubated with DHA. McA cells were pretreated with BSA or DHA/BSA for 1 hour, then labeled to steady state in the presence of BSA, DHA/BSA, DHA/ BSA plus $100 \mu \mathrm{M}$ DFX, or DHA/BSA plus $120 \mu \mathrm{M}$ vitamin E. Conditioned medium samples were then taken for the quantification of recovered total [ $\left.{ }^{35} \mathrm{~S}\right]-\mathrm{ApoB} 100$ (A), and for sucrose density gradient ultracentrifugation (B). After ultracentrifugation, the density fractions were collected and their ApoB100 contents analyzed as in Figure 1. ${ }^{*} P<0.01$ vs. DHA.
Because $\omega-3$ fatty acid-induced ApoB100 degradation preferentially targets large, buoyant lipoproteins (2), we next sought to determine whether these particles would be preferentially rescued by DFX or vitamin E and appear in the medium. Sucrose density gradient ultracentrifugation of media indicated that under control conditions, $63 \%$ of the total ApoB100 that was secreted by McA cells was in the form of VLDL (i.e., in the $d \leq 1.006$ fraction; Figure 3B). DHA incubation reduced VLDL-ApoB100 secretion to only $34 \%$ of the total ApoB100 output from these cells. Importantly, DFX or vitamin E restored the fraction of the total ApoB100 that was secreted as VLDL to $60 \%$ or $63 \%$, respectively; i.e., essentially the control value (Figure 3B). Thus, inhibition of lipid peroxidation substantially prevented DHA-induced ApoB100 degradation and the preferential loss of large, buoyant ApoB-lipoproteins from conditioned media.

Lipid peroxidation regulates the secretion of Apolipoprotein $E$ (ApoE), but not its degradation. We have previously reported that DHA or EPA decreased the recovery of metabolically labeled ApoE from rat hepatic cells (2). To determine whether the basis for this was the same as for the effects of DHA or EPA on ApoB100 secretion, i.e., an increase in degradation related to cellular lipid peroxide levels, pulse-chase studies similar to those described above were performed in rat primary hepatocytes and McA cells, with similar results obtained from either cell type.

As shown in Figure 4A, the recoveries of total labeled ApoE (cell+medium) from rat primary hepatocytes were not significantly different among the treatments (OA, DHA, and DHA+DFX), which is consistent with the reports that ApoE is relatively resistant to intracellular degradation (e.g., ref. 43). Although DFX did not affect ApoE degradation, the inhibition of lipid peroxidation increased the fraction of the cellular pool of ApoE that was secreted (Figure $4 \mathrm{~B})$. An interpretation of these results is given in the Discussion.

Common dietary PUFAs also promote intracellular lipid peroxidation and induce hepatic ApoB100 degradation. Like marine $\omega-3$ fatty acids, common dietary PUFAs from nonmarine sources are good substrates for lipid peroxidation and have long been known to lower plasma concentrations of ApoB-lipoproteins in vivo (reviewed in ref. 44). To determine whether these common PUFAs provoke lipid peroxide-dependent degradation of newly synthesized ApoB100, we conducted pulse-chase experiments with McA cells incubated in the presence of the following fatty acids complexed to BSA: myristic acid (MA; 14:0; a saturated fatty acid that is not subject to peroxidation because of the absence of double bonds), oleic acid (OA; 18:1; an $\omega-6$ monounsaturated fatty acid that is relatively resistant but not impervious to peroxidation), linolenic acid (LNA; 18:3; a nonmarine $\omega$-3 PUFA), arachidonic acid (AA; 20:4; an $\omega-6$ PUFA), or DHA. Control cells were incubated under the same conditions, but in the presence of BSA without any exogenously added fatty acid.

As shown in Figure 5A, cellular levels of TBARSs after these treatments were higher when the exogenous fatty acid was polyunsaturated, independent of the position of the double bonds. The recoveries of total $\left[{ }^{35} \mathrm{~S}\right]$-ApoB100 are summarized in Figure 5B and show a general inverse relationship to the TBARS results. To statistically analyze the relationship between TBARSs and ApoB100 recovery, a regression analysis was performed using the data from Figure 2 and Figure 5, A and B, in which experiments were conducted under similar conditions with the same cell type, as well as the data from
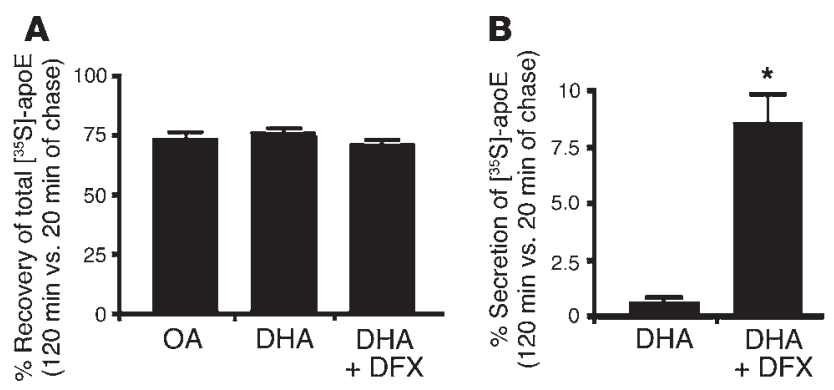

\section{Figure 4}

Lipid peroxidation regulates the secretion of ApoE, but not its degradation. Rat primary hepatocytes were maintained overnight in modified Waymouth's medium (Figure 1) and then treated with OA/BSA, DHA/ $\mathrm{BSA}$, or DHA/BSA plus $100 \mu \mathrm{M}$ DFX in a pulse-chase protocol similar to that used for Figure 2B. Cell lysate and medium samples were collected at 20 and 120 minutes of the chase period, and the recovery of total (cell + medium) labeled ApoE $(\mathbf{A})$ or the fractional secretion of labeled ApoE (B) was determined. ${ }^{*} P<0.004$ vs. DHA. 
A

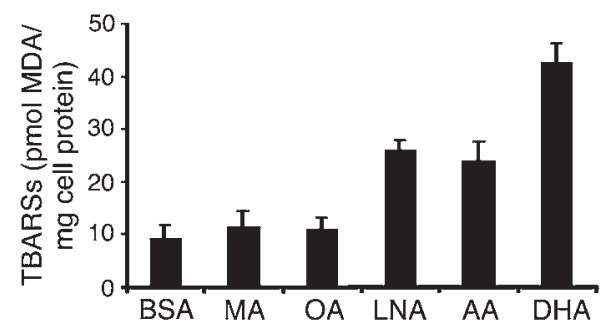

B

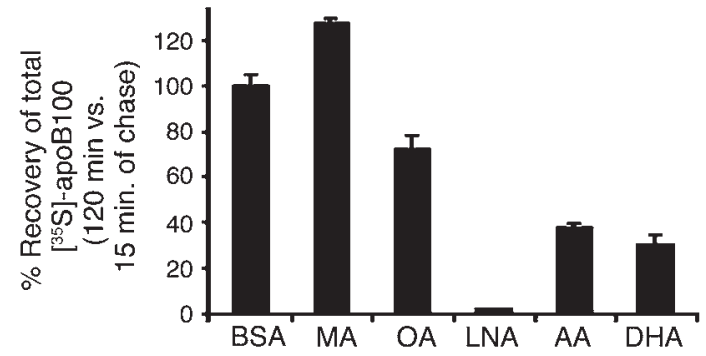

C

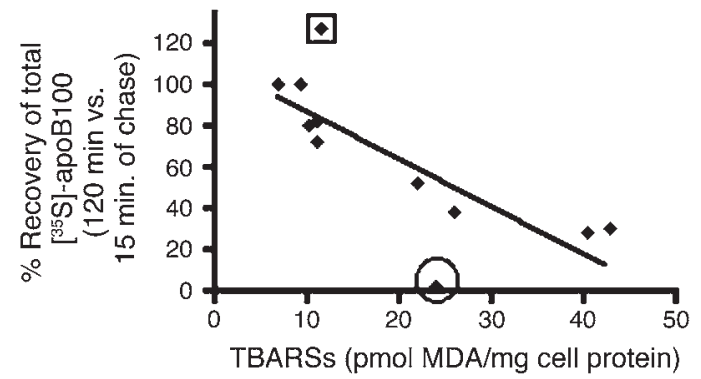

an experiment using $o$-phenanthroline $(60 \mu \mathrm{g} / \mathrm{ml})$, a weak iron chelator that decreases fish oil-induced hepatic ApoB100 degradation (45) and TBARS levels (M. Pan and E.A. Fisher, unpublished data). As shown in Figure 5C, there was a significant inverse correlation between TBARSs and ApoB100 recovery $(r=-0.78, P<0.005)$.

There are two conspicuous outliers, however, namely MA and LNA. If these outliers were not included in the analysis, the $r$ value improved to $-0.93(P<0.002)$. For MA, we have previously shown that this fatty acid has a depressive effect on ApoB100 degradation related to decreased recruitment of triglycerides to form smaller VLDL particles (which are not good substrates for PERPP) (4). This may explain why the ApoB100 recovery is higher than would be expected from the TBARS data alone. For LNA, although there is an increase in TBARSs over control, they are lower than in DHA-treated cells, yet there is virtually no recovery of ApoB100. Nevertheless, as with DHA, cotreatment with DFX blocked the effects of LNA (or any of the PUFAs) on ApoB100 recovery and TBARSs (data not shown).

\section{Figure 6}

DHA-induced ApoB100 degradation in primary rat hepatocytes also requires the increased generation of lipid peroxides. Primary rat hepatocytes were maintained overnight in modified Waymouth's medium (Figure 1). Two different treatment protocols were subsequently followed: (A) Cells were incubated for 1 hour with either BSA or DHA/ BSA, followed by 3 hours of incubation with BSA, DHA/BSA, DHA/BSA plus $100 \mu \mathrm{M}$ DFX, or DHA/BSA plus $200 \mu \mathrm{M}$ vitamin E. Shown are the TBARS assay results. ${ }^{\star} P<0.01$ vs. DHA. (B) Cells were pretreated with BSA or DHA/BSA for 2 hours, followed by pulse labeling and a chase period up to 120 minutes. DFX or vitamin $E$ was added as in Figure 2B. The recovery of total labeled ApoB100 was determined as in Figure 1. ${ }^{\star} P<0.01$ vs. DHA.

\section{Figure 5}

Nonmarine PUFAs promote intracellular lipid peroxidation, which inversely correlates with ApoB100 recovery. (A) McA cells were incubated for a total of 4 hours with fatty acids of various degrees of length and saturation and then taken for the measurement of TBARSs. (B) McA cells were pretreated with the fatty acids for 2 hours and were then subjected to a pulse-chase study as for Figure 2B. Displayed are the total labeled ApoB100 recovery data, determined as in Figure 1. (C) Regression analysis of the relationship between cellular TBARSs and total ApoB100 recovery. See the text for details concerning the data included in this analysis. The two outliers, MA and LNA, are indicated by a square and a circle, respectively.

Although the results indicate a common mechanism among conventional and marine PUFAs, in which iron-dependent lipid peroxidation promotes the degradation of newly synthesized ApoB100, the data with LNA suggest, among other possibilities, that all components of TBARSs may not be entirely equivalent in this process or that different PUFAs may undergo lipid peroxidation in specific locations where ApoB100 varies in its susceptibility to their effects.

Lipid peroxidation regulates both DHA-induced and basal ApoB100 degradation in primary rodent hepatocytes. Though McA cells have many features in common with primary rat hepatocytes $(46,47)$, to avoid potential artifacts from the use of a transformed cell line, we directly tested in primary rat hepatocytes the role of lipid peroxidation in ApoB100 degradation and secretion. As shown in Figure 6, A and B, in the DHA-treated cells, TBARSs increased fivefold over the level in the BSA-treated cells, whereas ApoB100 recovery was only about $30 \%$ of the (basal) recovery of total $\left[{ }^{35} \mathrm{~S}\right]-\mathrm{ApoB} 100$ in BSA-treated cells.

Importantly, the induction of lipid peroxidation and the induction of ApoB100 degradation by DHA in primary rat hepatocytes were both essentially abolished by DFX (Figure 6, A and B). Vitamin E also produced substantial decreases in both cellular lipid peroxidation and ApoB100 degradation. Surprisingly, the recovery of ApoB100 after DHA+DFX was greater than control (BSA alone), implying that even in the basal state, lipid peroxidation may play a role in ApoB100 degradation (a point pursued below).

Similar to our findings in DHA-treated McA cells, there was substantial secretion of the ApoB100 rescued by DFX or vitamin E in

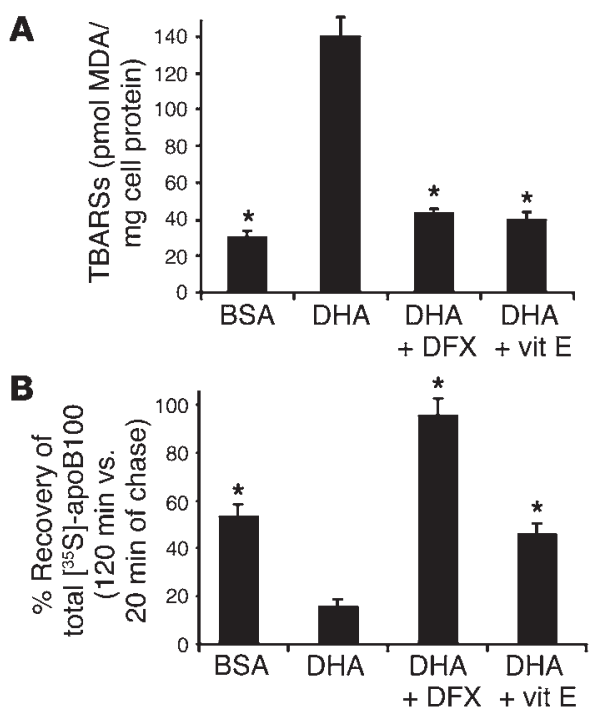



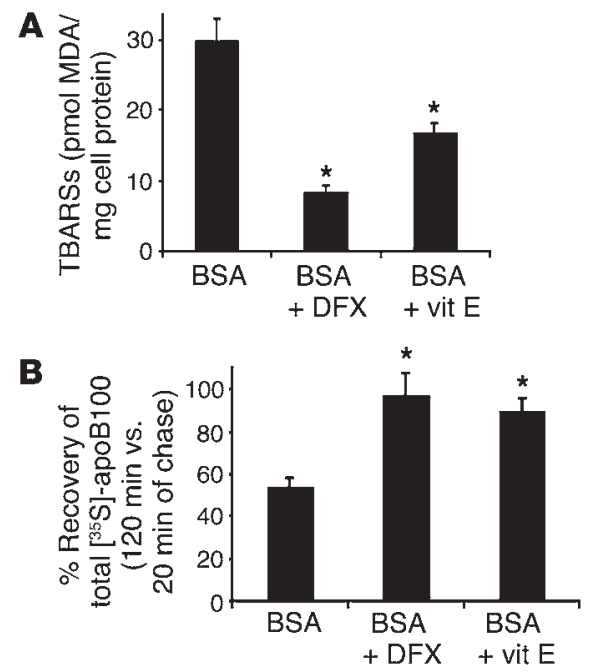

\section{Figure 7}

Lipid peroxides regulate basal ApoB100 degradation in primary rat hepatocytes. Primary rat hepatocytes were treated as in Figure 6, except that there was no DHA treatment. Shown are the data for TBARSs (A) and total labeled ApoB100 recovery (B). ${ }^{*} P<0.01$ vs. BSA.

DHA-treated primary rat hepatocytes (percentage of initial labeled cellular ApoB100 that accumulated in the conditioned media: DHA, $2.0 \% \pm 0.3 \%$; DHA+DFX, 29\% $\pm 4 \%$; DHA+vitamin E, $14 \% \pm 2 \%$; $P<0.001$, DHA+DFX or DHA+vitamin E vs. DHA). The ability of compounds with antioxidant activity to coordinately reduce lipid peroxide generation and increase ApoB 100 recovery and secretion was also observed in primary mouse hepatocytes (data not shown). Thus, in all three cell types - primary rat and mouse hepatocytes and McA cells - DHA stimulated the degradation of newly synthesized ApoB100 and inhibited ApoB-lipoprotein secretion through a lipid peroxide-dependent mechanism.

The relatively high level of TBARSs in BSA-treated hepatocytes and the ability of DFX+DHA cotreatment to increase ApoB100 recovery over control both suggested that basal (i.e., in the absence of exogenous fatty acids) ApoB100 degradation in normal, nonfasted liver cells may have regulatory features similar to PUFA-induced PERPP.

To provide direct support for this suggestion, rat primary hepatocytes in basal medium were subjected to pulse-chase studies in the presence or absence of DFX or vitamin E. As shown in Figure 7, A and B, DFX treatment substantially reduced TBARS levels and ApoB100 degradation. In the basal state (BSA treatment), ApoB100 degradation was about 46\%, consistent with the initial report of about $50 \%$ ApoB100 degradation in rat primary hepatocytes (48). In addition, the secretion of ApoB 100 increased from $27 \%$ to $46 \%$ of the initially labeled cellular pool. Vitamin E treatment resulted in a similar pattern: reduction of TBARSs, a decrease in ApoB100 degradation, and an increase in ApoB100 secretion to 40\%. These observations were also reproduced with mouse primary hepatocytes (data not shown). It should also be noted that in BSA-treated rat primary hepatocytes, treatment with the specific proteasome inhibitor lactacystin failed to increase the recovery of labeled ApoB100 (data not shown), as has also been seen in previous studies (49), making it unlikely that ERAD is a major contributor to basal ApoB100 degradation in primary hepatocytes under the metabolic conditions of our experiments.
The above results support a role for lipid peroxide regulation of PERPP in the degradation of ApoB100 under basal conditions. Consistent with this role is the considerable difference in the basal level of ApoB100 degradation between McA cells and rat primary hepatocytes (compare Figure 2 with Figure 7). A likely basis for this is the TBARS content of primary hepatocytes compared with that of McA cells (McA, $9.3 \mathrm{pmol}$ malondialdehyde (MDA)/mg cell protein; primary hepatocytes, $30 \mathrm{pmol} \mathrm{MDA} / \mathrm{mg}$ cell protein). This inverse correlation between the levels of basal degradation and lipid peroxidation is similar to the results for $\omega-3$ fatty acid stimulation of PERPP (Figure 5).

Infusion of DHA into living mice increases hepatic lipid peroxide content and reduces VLDL-ApoB100 secretion in vivo. We sought to determine whether the mechanistic links we found in vitro among PUFA, lipid peroxidation, and ApoB100 degradation are also functionally important in vivo. To quickly produce effects of DHA $(0.2 \mathrm{mM}$ complexed to $0.04 \mathrm{mM} \mathrm{BSA}$ ) in vivo under controlled conditions, we made minor modifications of a previously established protocol for infusing FA/BSA complexes into the mouse jugular vein (see Methods; also Y.-L. Zhang and H.N. Ginsberg, unpublished data). Infusion of $0.2 \mathrm{mM}$ OA (complexed to $0.04 \mathrm{mM} \mathrm{BSA}$ ) was used as control. At this concentration, OA does not affect hepatic VLDL-ApoB100 production compared with saline-infused controls (Y.-L. Zhang and H.N. Ginsberg, unpublished data), consistent with the lack of stimulation by OA of ApoB100 secretion in rat primary hepatocytes (50).

After 6 hours of infusion, each mouse was injected intravenously with a bolus of $\left.{ }^{35} \mathrm{~S}\right]-\mathrm{Met} / \mathrm{Cys}$, followed immediately by Triton WR1339, a reagent that blocks clearance of ApoB-lipoproteins, thereby allowing direct measurements of hepatic secretion of labeled ApoB100 in vivo (51). One hour after radioisotope injection, blood samples were collected and assayed for plasma $\left[{ }^{35} \mathrm{~S}\right]$ ApoB100, which reflects VLDL-[ ${ }^{35}$ S]-ApoB100 secretion by the liver according to our published study (52). The lipid peroxide levels of the liver homogenate were also measured.

The lipid peroxide levels in the livers of control mice (OAinfused) were statistically indistinguishable from hepatic levels in mice without any infusion treatment (data not shown). However, consistent with our data in vitro, as shown in Figure 8A, DHA infusion was associated with a substantial (>40\%) elevation in hepatic lipid peroxide content. Importantly, DHA treatment in vivo also produced a significant $(>30 \%)$ decrease in the secretion of newly synthesized ApoB100 (Figure 8B). Thus, as we found in vitro, administration of DHA increases hepatic lipid peroxidation and decreases the secretion of VLDL-ApoB100.

\section{Discussion}

We have previously shown that marine $\omega$ - 3 fatty acids, which are clinically useful hypolipidemic agents (53), stimulate a post-ER presecretory proteolysis (PERPP) of newly synthesized ApoB100 that is not mediated by either the proteasome or the lysosome (2). In the current study, we have shown that these fatty acids stimulate this form of ApoB100 degradation through their ability to increase the cellular content of lipid peroxidation products. Moreover, this novel link between cellular oxidant stress and presecretory degradation of ApoB100 was not confined to marine $\omega-3$ fatty acids: our studies also show that additional species of PUFAs stimulate the loss of nascent ApoB100, and that this loss occurs in a lipid peroxide-dependent manner.

The link between oxidant stress and ApoB100 degradation was further strengthened by experiments in which the addition of 
A

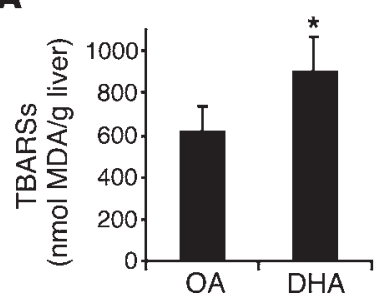

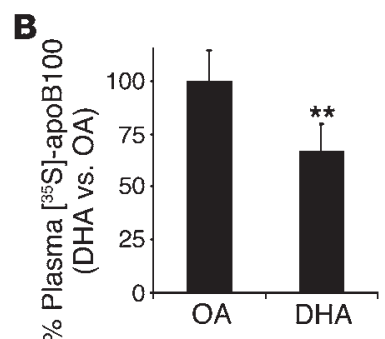

Figure 8

DHA infusion into living mice increases hepatic lipid peroxidation and reduces VLDL-ApoB100 secretion. C57BL/6J mice were infused with $\mathrm{DHA} / \mathrm{BSA}$ or OA/BSA for 6 hours. The mice were then injected via the jugular vein with $\left.{ }^{35} \mathrm{~S}\right]-M e t / C y s$ together with Triton WR1339. After 60 minutes, liver homogenate and plasma samples were prepared. (A) Lipid peroxidation levels in the liver homogenate samples. ${ }^{*} P<0.03$ vs. OA. (B) Plasma [ ${ }^{35}$ S]-ApoB100, which reflects hepatic production of VLDL-[ $\left.{ }^{35} \mathrm{~S}\right]-A$-ABoB100, was determined by SDS-PAGE/fluorography. ${ }^{\star *} P<0.003$ vs. OA.

agents with antioxidant activities, such as DFX or vitamin E, in the presence of marine $\omega-3$ or other PUFAs, substantially reduced degradation, resulting in increased secretion of VLDL. The effects of DFX, an iron chelator that inhibits iron-dependent lipid peroxidation, may explain the reported ability of $o$-phenanthroline, a cell-permeable reagent that also chelates iron, to decrease DHAstimulated ApoB100 degradation and increase VLDL secretion (45), especially because $o$-phenanthroline lowers the TBARS contents of rat hepatic cells incubated with DHA (M. Pan and E.A. Fisher, unpublished data; and Figure 5C).

Quite striking was the finding that lipid peroxidation within primary cultured hepatocytes promoted degradation and inhibited secretion of ApoB100 even in the basal state, i.e., in the absence of exogenous sources of unsaturated fatty acids. Since its discovery in rat primary hepatocytes (48), basal degradation of ApoB100 has been a subject of investigation and speculation. While ERAD of ApoB100 has been found in hepatic lipid-deficiency states in vitro and in vivo $(1,3)$, the lack of an effect of proteasome inhibitors on PERPP (2) or on basal degradation of ApoB100 in the present and published studies (49) implicates PERPP or a PERPP-like pathway. Overall, then, our results from studies both in vitro and in vivo indicate a broad and central role for lipid peroxidation and oxidant stress in the physiological control by PERPP of ApoB-lipoprotein secretion from hepatocytes under a broad range of metabolic conditions.

Regulatory effects of oxidants have recently received substantial attention. Some growth factors (e.g., EGF [ref. 54] and insulin [ref. 55]) appear to use hydrogen peroxide as a second messenger to mediate key downstream effects, including PI3K activation, although a link to other oxidant stress molecules, such as lipid peroxides, has not been examined. To investigate whether hydrogen peroxide had a specific role in our system, we performed pulsechase studies in the presence of exogenous catalase (up to $0.015 \%$, $\mathrm{wt} / \mathrm{vol}$, a concentration twice that needed to deplete hepatic cells of hydrogen peroxide [ref. 55]). There was no effect on ApoB100 recovery from DHA-treated cells (M. Pan et al., unpublished data), suggesting that another iron-dependent oxidant is important in catalyzing the lipid peroxidation required for the stimulation of ApoB100 degradation. Several signaling paths could exist between lipid peroxidation products and ApoB100 destruction by PERPP that, for example, ultimately alter intracellular trafficking of newly assembled ApoB-lipoproteins to a yet unidentified degradation compartment or protease(s). This would be consistent with our previous demonstration that inhibition of PI3K reduces DHAinduced ApoB100 degradation (2).

Our current results also suggest another possibility, namely, direct oxidative damage to ApoB100, which could promote either enzymatic or nonenzymatic degradation (56). This would certainly be plausible if the lipid peroxides were part of the nascent lipoproteins, resulting in a high local concentration of damaging agents. This would also be consistent with the recent finding (57) in rat liver that microsome-associated ApoB100 was associated with the ironbinding protein ferritin, which could serve, perhaps, a biological role akin to the protective effects of DFX. Another potential "protector" of ApoB100 is ApoE, which associates with ApoB-lipoproteins within the secretory pathway (58), has antioxidant activities (59, 60 ), is relatively resistant to intracellular degradation (43), and can promote hepatic VLDL production (e.g., ref. 61). Our findings that cellular lipid peroxide levels regulated ApoE secretion, but not degradation (Figure 4), suggest that whatever protection ApoE affords to ApoB100 can be overcome and the nascent VLDL diverted from the secretory pathway. Presumably, ApoE would still not share the same degradative fate as ApoB100, either because of its aforementioned resistance to proteolysis or because, as a surface exchangeable protein, it becomes uncoupled from the intracellular itinerary of ApoB100 (consistent with the aggregation data, described just below). We are currently investigating these possibilities.

Generally, oxidized proteins in mammalian cells can undergo chemical fragmentation or form large aggregates owing to covalent cross-linking and increased surface hydrophobicity (56). Indeed, preliminary data indicate the lipid peroxidation-dependent formation of high-molecular weight aggregates containing oxidized ApoB100 (but not ApoE) in cells incubated with DHA (M. Pan et al., unpublished data). Relevant to the notion that intracellular oxidative damage of ApoB100 directly or indirectly promotes degradation is the abundant literature that the extracellular oxidation of purified LDL in vitro often causes the nonenzymatic breakdown of ApoB100 (62) and can facilitate the enzymatic cleavage of ApoB100 by secreted proteases such as leukocyte elastase or MMPs (63).

Our finding that infusion of DHA in mice increased hepatic lipid peroxide content is consistent with a large body of literature, primarily from studies of animals fed on diets enriched in PUFAs (45, 64-67). For example, feeding rats on diets enriched in EPA, DHA, or LNA increased hepatic lipid peroxidation and the breakdown of vitamin E (64). In another rat-feeding study, the ingestion of DHA-containing oils decreased triglyceride concentrations in the plasma and liver at the same time that hepatic levels of lipid hydroperoxides and TBARSs rose and vitamin E fell (65). In mice fed fish oil-enriched diets for 6 months, transcript profiling of the liver revealed increased expression of genes encoding antioxidantrelated proteins, such as several glutathione transferases, uncoupling protein 2, and Mn-superoxide dismutase, consistent with a defensive response against increased oxidant stress (68). It will be of interest, then, in our future studies to determine whether increasing the capacity to oppose oxidant stress by pre- or coadministration of DFX or vitamin E will attenuate hepatic lipid peroxide content and promote VLDL-ApoB100 secretion, either in the context of a DHA infusion or in the basal state.

A number of clinical studies indirectly support the regulation of ApoB-lipoprotein production by oxidant stress. For example, after 3-8 weeks of dietary supplementation with EPA and DHA, 
healthy volunteers showed increased plasma concentrations of lipid peroxides along with deceased triglycerides and LDL (69-71). The classic dietary studies of Hegsted, Keys, Ahrens, and others (reviewed in ref. 44; see also refs. 72-74) have consistently shown that PUFAs had hypolipidemic effects and saturated fatty acids had hyperlipidemic effects. There is evidence that these effects were partially attributable to induced changes in hepatic LDL receptor activity affecting lipoprotein clearance (75). The current results, taken with our recent report (4), show that ApoB100 production and degradation in hepatic cells are inversely and directly related, respectively, to the degree of polyunsaturation of the supplied fatty acids. Thus, a varying degree of hepatic oxidant stress induced by different fatty acids may be another major mechanistic basis for the classic dietary observations.

As noted above, a striking finding of the present study is that lipid peroxidation played a substantial role in regulating the output of ApoB100 from hepatocytes even in the absence of added PUFA. Thus, decreased lipid peroxidation products or enhanced antioxidants in the liver would be expected to increase net hepatic ApoB100 production, and thereby to elevate plasma levels of VLDL or LDL. This line of reasoning provides an attractive explanation for the pathophysiology of a spontaneously mutant mouse proposed to be a model for the human disease Familial Combined Hypercholesterolemia $(76,77)$. The murine combined hyperlipidemia is characterized by increased secretion of triglyceride-rich lipoproteins and elevated plasma concentrations of triglyceride, cholesterol, and ApoB100. The mutation results in low thioredoxin-interacting protein (TxnIP) levels (77). Because TxnIP inhibits thioredoxin, a major endogenous cellular antioxidant, the mutation should increase the hepatic antioxidant capacity, which we would associate with lipoprotein overproduction and hyperlipidemia. Other situations in which our findings may provide a mechanistic basis include human dyslipidemias - particularly those associated with insulin resistance, since insulin has also been shown to regulate a PERPP-like ApoB100-degradative process $(1,2)$ - and the decreased hepatic secretion of ApoB100 in mice with phospholipid transfer protein deficiency. This deficiency results in increased hepatic levels of TBARS and decreased hepatic levels of vitamin E (78).

Manipulation of oxidation in humans for the purpose of preventing cardiovascular disease has received substantial attention and effort. Administration of antioxidants, such as vitamin E, has generally lessened arterial lesions in animal models of atherosclerosis but has had no consistent benefit (e.g., refs. 29, 30), and has resulted possibly in occasional harm $(28,79)$, in the authentic human disease. Our current results imply that potential benefits of antioxidants on human arterial lesion development might be counteracted by potentially harmful alterations in ApoB100 metabolism within the liver. Thus, a search for antioxidants that either do not enter the liver or do not affect hepatic lipid peroxidation might be warranted. In addition, agents that could transport oxidized or oxidizable material from the arterial wall, where it may be harmful, to the liver, where it may be beneficial, may be desirable (80).

In conclusion, our data firmly establish a novel link between cellular lipid peroxidation and oxidant stress with the destruction of newly synthesized ApoB100 via PERPP. These results have wide implications. The basal turnover of ApoB100, the origins of hyperlipidemias that involve ApoB100 overproduction, the hypolipidemic actions of fish oils and other conventional PUFAs, the relationship between insulin resistance and dyslipidemia, and perhaps the clinical utility of antioxidants are all phenomena on which lipid peroxidation and PERPP may exert significant regulatory impact.

\section{Methods}

Unless otherwise indicated, the materials and methods closely followed published protocols used in the prior work by us $(2,4,50$, 81 ) and others (82-85). All animal procedures were approved by the Mount Sinai Animal Care Committee.

Cell culture. Rat hepatoma McA cells, obtained from American Type Culture Collection (Manassas, Virginia, USA), were grown in six-well plates or P100 cell-culture dishes coated with type I collagen from rat tails. Note that of the two apolipoprotein B species secreted by rodent liver, ApoB100 and ApoB48, this clone of cells produces predominantly ApoB100 (4).

Mouse or rat primary hepatocytes were isolated and cultured as described (50, 82).

Metabolic labeling of ApoB100 or ApoE. Two protocols were followed: pulse chase or continuous labeling. At the beginning of a pulse-chase study, cells were preincubated for 60 minutes in medium containing either $0.12 \mathrm{mM}$ BSA (fatty acid-free) or 0.6 $\mathrm{mM}$ fatty acid (complexed to $0.12 \mathrm{mM}$ BSA), except for AA, which was administered at a concentration of $175 \mu \mathrm{M}$ to avoid cellular toxicity. DMEM (for McA cells) was also supplemented with $0.5 \%$ FBS, $0.5 \%$ horse serum, $1 \%$ L-glutamine, and $1 \%$ penicillin/streptomycin. Waymouth's medium (for primary hepatocytes) was supplemented with $0.1 \mathrm{nM}$ insulin and $0.2 \% \mathrm{BSA}$. The corresponding BSA and FA/BSA treatments were continued throughout the experiments. After this preincubation, there was a 60 -minute incubation in Met/Cys-free medium. To pulse-label the cells, they were then incubated in Met/Cys-free medium supplemented with 200 $\mu \mathrm{Ci}\left[{ }^{35} \mathrm{~S}\right]-\mathrm{Met} / \mathrm{Cys}$ per milliliter of medium for 15 minutes (McA cells), 20 minutes (rat primary hepatocytes), or 30 minutes (mouse primary hepatocytes). The chase period was begun by removal of the labeling medium, washing of the cell monolayer with cold PBS, and addition of medium at $37^{\circ} \mathrm{C}$ without radioisotopes but supplemented with an excess of unlabeled Met/Cys (10 mM Met, $3 \mathrm{mM}$ Cys). The initial chase points were identical to the labeling times. These labeling and initial chase periods were designed to highly label the ApoB100 pool and to sample the peak incorporation of isotope into ApoB100 for each cell type (data not shown).

At the time points indicated in Results, medium samples were collected, and cell monolayers were washed twice with cold PBS and lysed in $10 \mathrm{mM}$ PBS (pH 7.4), $125 \mathrm{mM} \mathrm{NaCl}, 36 \mathrm{mM}$ lithium dodecyl sulfate, $24 \mathrm{mM}$ deoxycholate, and 1\% Triton X-100. Both media and cell lysate samples were supplemented with protease inhibitors. $\left[{ }^{35} \mathrm{~S}\right]$-labeled Apoproteins were immunoprecipitated with rabbit polyclonal antiserum to rat ApoB100 or goat polyclonal antiserum to mouse ApoB100 (developed in the authors' laboratories), or rabbit polyclonal antiserum to rat ApoE (a generous gift of Janet D. Sparks) (2, 50, 81). Quantification was performed by SDS-PAGE (4\% polyacrylamide, $0.1 \%$ SDS, $1 \mathrm{M}$ urea), fluorography, and densitometry, as we previously described $(2,50,81)$. Total protein synthesis was measured by determination of the trichloroacetic acid-precipitable radioactivity in aliquots of cell lysates and conditioned media (2).

For steady-state labeling, an identical protocol was followed, except that the labeling lasted at least 2 hours and there was no chase period. In some experiments, cells were treated with a variety of compounds, including enzyme inhibitors, calcium perturbants, and inhibitors of lipid peroxidation, as indicated in Results. Also, 
in some experiments, sucrose-gradient analysis of the buoyant densities of the lipoproteins in conditioned medium was carried out according to a modification of the protocol of Stillemark et al. (83). The sucrose gradient was formed by layering of $1.95 \mathrm{ml}$ of $49 \%$ sucrose, $1.95 \mathrm{ml}$ of $25 \%$ sucrose, $4.75 \mathrm{ml}$ of sample in $12.5 \%$ sucrose, and $2.85 \mathrm{ml}$ of PBS. All solutions in the gradient contained protease inhibitors as well as $150 \mathrm{mM} \mathrm{NaCl}, 50 \mu \mathrm{M}$ of the synthetic antioxidant butylated hydroxytoluene (BHT), and $50 \mathrm{mM}$ PBS (pH 7.4). The gradients were ultracentrifuged at 210,000 $\mathrm{g}$ in a swinging-bucket SW41 rotor (Beckman Coulter, Fullerton, California, USA) for 65 hours at $10^{\circ} \mathrm{C}$, then unloaded into 12 fractions. Labeled ApoB100 was recovered from each fraction and analyzed by immunoprecipitation, SDS-PAGE, and fluorography, as above.

Lipid peroxidation assays. The content of lipid peroxides in cultured cell lysate was determined by the classical method of measuring TBARSs (84). Briefly, cells (in a P100 dish) were preincubated for 1 hour in the presence of BSA or FA/BSA (in the appropriate medium as described above in "Metabolic labeling of ApoB100 or ApoE"), followed by a 3-hour incubation in phenol red-free medium (to avoid interference with absorption at $532 \mathrm{~nm}$ ), in the continued presence of BSA or FA/BSA. Some wells were supplemented with the antioxidants indicated in Results. Cells were then washed twice in cold PBS, scraped off the dishes, and then suspended in $12 \mathrm{ml}$ of PBS supplemented with $150 \mu \mathrm{M}$ BHT (to prevent any further lipid peroxide formation). After centrifugation at $2,000 \mathrm{~g}$ for 5 minutes, the supernatant was discarded, and the cells were resuspended in $150 \mu \mathrm{l}$ of PBS with BHT. The cell suspension $(100 \mu \mathrm{l})$ was combined with freshly made $50 \mu \mathrm{l} 30 \%$ trichloroacetic acid, $0.75 \%$ thiobarbituric acid, and $0.5 \mathrm{~N} \mathrm{HCl}$, and incubated for 15 minutes at $100^{\circ} \mathrm{C}$. The reaction mixture was then centrifuged for 8 minutes at $13,500 \mathrm{~g}$. The absorbance of the supernatant was measured at $532 \mathrm{~nm}$ (the contribution of absorbance by a peak at less than $532 \mathrm{~nm}$ was deducted). The concentration of MDA equivalents was calculated using an extinction coefficient of $1.56 \times 10^{5} \mathrm{M}^{-1} \mathrm{~cm}^{-1}$. The rest of the cells were used for protein determination using the DC protein assay kit (Bio-Rad, Hercules, California, USA). Similar assays were performed on stock solutions of FA/BSA complexes to detect pre-existing lipid peroxidation, or on medium placed in wells without cells to detect lipid peroxidation independent of cellular metabolism. In both cases, there were no TBARSs above background.

Fatty acid infusion in vivo. Male C57BL/6J mice (12-14 weeks of age), maintained on a chow diet, were anesthetized with ketamine/ xylazine. Silicone rubber tubing filled with saline was inserted into the jugular vein, and the outer portion of the tubing was tunneled subcutaneously so that its exit was at the nape of the neck, where it was inaccessible to the mouse. The tubing was closed at the end with a metal stopper. The mice were allowed to recover for 12-15 hours before the infusion experiment.

On the morning of the experiment, food was removed, and the silicone tubing was connected to a Harvard Compact Infusion Pump (Harvard Apparatus, Holliston, Massachusetts, USA). The infusate was freshly prepared and contained a final concentration of $0.2 \mathrm{mM}$ fatty acid (DHA or OA), complexed to $0.04 \mathrm{mM}$ BSA, in buffered saline. The infusion line ran inside a tether and through a swivel to allow for free movement of the mice. The tubing was wrapped with black plastic to shield the fatty acids from light. A modified TBARS assay (see below) was performed before and after the infusion, and no reactive oxygen species were detected in either the DHA or the OA infusates. The infusion was carried out at the rate of $3 \mu \mathrm{l} / \mathrm{min}$ for 6 hours. Blood samples were obtained from the retro-orbital plexus before and after the 6-hour infusion.

After completion of the infusion, mice were injected intravenously with $\left[{ }^{35} \mathrm{~S}\right]-\mathrm{Met} / \mathrm{Cys}(200 \mu \mathrm{Ci}$ per mouse, in $100 \mu \mathrm{l} 0.9 \%$ $\mathrm{NaCl}$ ) together with $500 \mathrm{mg}$ Triton WR1339 per kilogram body weight $(15 \% \mathrm{vol} / \mathrm{vol}$ stock diluted in $0.9 \% \mathrm{NaCl})$. One hour after radioisotope injection, blood samples $(80 \mu \mathrm{l})$ were collected and plasma was separated immediately. The animals were sacrificed upon completion of the experiment. The liver was flushed with $0.9 \% \mathrm{NaCl}$ and freshly homogenized with $1.15 \% \mathrm{KCl} / 0.2 \mathrm{mg} \mathrm{BHT}$ per $\mathrm{ml}(9 \mathrm{ml} / \mathrm{g}$ tissue). Both the plasma and the liver homogenate were quick-frozen in liquid $\mathrm{N}_{2}$ and stored at $-80^{\circ} \mathrm{C}$ until further use. To determine the plasma accumulation of [ $\left.{ }^{35} \mathrm{~S}\right]$-labeled VLDLApoB100 after Triton injection, at the indicated time points, plasma was collected, mixed with equal volume of sample buffer, and heated at $95^{\circ} \mathrm{C}$ for 5 minutes prior to SDS-PAGE, fluorography, and densitometry (52). The content of lipid peroxides in liver homogenates was measured according to the method of Ohkawa et al. (85) using tetraethoxypropane as an external standard.

Statistical analyses. For any treatment, two to three wells per experiment were studied and the entire experiment was conducted at least three times. Data are typically expressed as mean \pm SD. Statistical differences and linear regression were analyzed by GraphPad Prism software (GraphPad Software Inc., San Diego, California, USA). Data between two groups were analyzed by unpaired, twotailed $t$ tests, and among three groups by ANOVA and the Dunnett post-test for multiple comparisons. A $P$ value of less than 0.05 was considered significant.

\section{Acknowledgments}

We thank Cristina Villagra for technical assistance and Barry Goldstein (Thomas Jefferson University) for advice on the catalase experiment. These studies were supported by grants from the NIH (HL58541 to E.A. Fisher; AA012757 to A.I. Cederbaum; HL55368 and HL73030 to H.N. Ginsberg; HL56984 to K.J. Williams) and the American Diabetes Association (RA-81 to K.J. Williams).

Received for publication June 13, 2003, and accepted in revised form January 29, 2004.

Address correspondence to: Edward A. Fisher, TH-451, New York University School of Medicine, 550 First Avenue, New York, New York 10016, USA. Phone: (212) 263-6631; Fax: (212) 263-6632; E-mail: edward.fisher@med.nyu.edu. Or to: Kevin Jon Williams, Thomas Jefferson University, Alumni Hall 349, 1020 Locust St., Philadelphia, Pennsylvania 19107, USA. Phone: (215) 503-0987; Fax: (215) 923-7932; E-mail: K_Williams@mail.jci.tju.edu.

\footnotetext{
1. Fisher, E.A., and Ginsberg, H.N. 2002. Complexity in the secretory pathway: the assembly and secretion of apolipoprotein B-containing lipoproteins. J. Biol. Chem. 277:17377-17380.

2. Fisher, E.A., et al. 2001. The triple threat to nascent apolipoprotein B. Evidence for multiple, distinct degradative pathways. J. Biol. Chem. 276:27855-27863.
}

3. Liang, J.S., et al. 2001. HIV protease inhibitors protect apolipoprotein $\mathrm{B}$ from degradation by the proteasome: a potential mechanism for protease inhibitor-induced hyperlipidemia. Nat. Med. 7:1327-1331.

4. Kummrow, E., Hussain, M.M., Pan, M., Marsh, J.B., and Fisher, E.A. 2002. Myristic acid increases dense lipoprotein secretion by inhibiting ApoB degradation and triglyceride recruitment. J. Lipid Res. 43:2155-2163.

5. Hegsted, D.M., McGandy, R.B., Myers, M.L., and Stare, F.J. 1965. Quantitative effects of dietary fat on serum cholesterol in man. Am. J. Clin. Nutr. 17:281-295.

6. Anderson, J.T., Grande, F., and Keys, A. 1976. Independence of the effects of cholesterol and degree 
of saturation of the fat in the diet on serum cholesterol in man. Am. J. Clin. Nutr. 29:1184-1189.

7. Phung, T.L., Roncone, A., Jensen, K.L., Sparks, C.E., and Sparks, J.D. 1997. Phosphoinositide 3-kinase activity is necessary for insulin-dependent inhibition of apolipoprotein B secretion by rat hepatocytes and localizes to the endoplasmic reticulum. J. Biol. Chem. 272:30693-30702.

8. Avramoglu, R.K., Qiu, W., and Adeli, K. 2003. Mechanisms of metabolic dyslipidemia in insulin resistant states: deregulation of hepatic and intestinal lipoprotein secretion. Front. Biosci. 8:D464-D476.

9. Yao, Z.M., and Vance, D.E. 1988. The active synthesis of phosphatidylcholine is required for very low density lipoprotein secretion from rat hepatocytes. J. Biol. Chem. 263:2998-3004.

10. Yeo, Y.K., and Holub, B.J. 1990. Influence of dietary fish oil on the relative synthesis of triacylglycerol and phospholipids in rat liver in vivo. Lipids. 25:811-814

11. Wu, X., Shang, A., Jiang, H., and Ginsberg, H.N. 1997. Demonstration of biphasic effects of docosahexaenoic acid on apolipoprotein B secretion in HepG2 cells. Arterioscler. Thromb. Vasc. Biol. 17:3347-3355.

12. Keller, H., et al. 1993. Fatty acids and retinoids control lipid metabolism through activation of peroxisome proliferator-activated receptor-retinoid X receptor heterodimers. Proc. Natl. Acad. Sci. U. S. A. 90:2160-2164.

13. Wong, S.H., Fisher, E.A., and Marsh, J.B. 1989. Effects of eicosapentaenoic and docosahexaenoic acids on Apoprotein B mRNA and secretion of very low density lipoprotein in HepG2 cells. Arteriosclerosis. 9:836-841.

14. Dallongeville, J., et al. 2001. Peroxisome proliferator-activated receptor alpha is not rate-limiting for the lipoprotein-lowering action of fish oil. J. Biol. Chem. 276:4634-4639.

15. Ventura, M.A., Woollett, L.A., and Spady, D.K. 1989. Dietary fish oil stimulates hepatic low density lipoprotein transport in the rat. J. Clin. Invest. 84:528-537.

16. Rumsey, S.C., Galeano, N.F., Lipschitz, B., and Deckelbaum, R.J. 1995. Oleate and other long chain fatty acids stimulate low density lipoprotein receptor activity by enhancing acyl coenzyme A: cholesterol acyltransferase activity and altering intracellular regulatory cholesterol pools in cultured cells. J. Biol. Chem. 270:10008-10016.

17. Jump, D.B. 2002. The biochemistry of n-3 polyunsaturated fatty acids. J. Biol. Chem. 277:8755-8758.

18. Bonin, A., and Khan, N.A. 2000. Regulation of calcium signalling by docosahexaenoic acid in human T-cells. Implication of CRAC channels. J. Lipid Res. 41:277-284.

19. Ferrier, G.R., Redondo, I., Zhu, J., and Murphy, M.G. 2002. Differential effects of docosahexaenoic acid on contractions and L-type Ca2+ current in adult cardiac myocytes. Cardiovasc. Res. 54:601-610.

20. Williams, K.J., Brocia, R.W., and Fisher, E.A. 1990. The unstirred water layer as a site of control of apolipoprotein B secretion. J. Biol. Chem. 265:16741-16744.

21. Gillian-Daniel, D.L., Bates, P.W., Tebon, A., and Attie, A.D. 2002. Endoplasmic reticulum localization of the low density lipoprotein receptor mediates presecretory degradation of apolipoprotein B. Proc. Natl. Acad. Sci. U. S. A. 99:4337-4342.

22. Twisk, J., et al. 2000. The role of the LDL receptor in apolipoprotein B secretion. J. Clin. Invest. 105:521-532.

23. Nossen, J.O., Rustan, A.C., and Drevon, C.A. 1987. Calcium-antagonists inhibit secretion of very-lowdensity lipoprotein from cultured rat hepatocytes. Biochem. J. 247:433-439.

24. Kwong, T.C., Sparks, J.D., Pryce, D.J., Cianci, J.F., and Sparks, C.E. 1989. Inhibition of apolipoprotein $B$ net synthesis and secretion from cultured rat hepatocytes by the calcium-channel blocker diltiazem. Biochem. J. 263:411-415.

25. Bjornsson, O.G., Bourgeois, C.S., and Gibbons, G.F. 1998. Varying very low-density lipoprotein secretion of rat hepatocytes by altering cellular levels of calcium and the activity of protein kinase C. Eur. J. Clin. Invest. 28:720-729.

26. Millar, J.S., Maugeais, C., Fuki, I.V., and Rader, D.J. 2002. Normal production rate of apolipoprotein B in LDL receptor-deficient mice. Arterioscler. Thromb. Vasc. Biol. 22:989-994.

27. Chirieac, D.V., Cianci, J., Collins, H.L., Sparks, J.D., and Sparks, C.E. 2002. Insulin suppression of VLDL Apo B secretion is not mediated by the LDL receptor. Biochem. Biophys. Res. Commun. 297:134-137.

28. Brown, B.G., et al. 2001. Simvastatin and niacin, antioxidant vitamins, or the combination for the prevention of coronary disease. N. Engl. J. Med. 345:1583-1592.

29. Steinberg, D., and Witztum, J.L. 2002. Is the oxidative modification hypothesis relevant to human atherosclerosis? Do the antioxidant trials conducted to date refute the hypothesis? Circulation. 105:2107-2111.

30. Collins, R., Peto, R., and Armitage, J. 2002. The MRC/BHF Heart Protection Study: preliminary results. Int. J. Clin. Pract. 56:53-56.

31. Ishibashi, S., et al. 1993. Hypercholesterolemia in low density lipoprotein receptor knockout mice and its reversal by adenovirus-mediated gene delivery. J. Clin. Invest. 92:883-893.

32. Larsson, S.L., Skogsberg, J., and Bjokegren, J. 2004. The low density lipoprotein receptor prevents secretion of dense ApoB100-containing lipoproteins from the liver. J. Biol. Chem. 279:831-836.

33. Karim, S., Habib, A., Levy-Toledano, S., and Maclouf, J. 1996. Cyclooxygenase- 1 and -2 of endothelial cells utilize exogenous or endogenous arachidonic acid for transcellular production of thromboxane. J. Biol. Chem. 271:12042-12048.

34. Hamill, O.P., and McBride, D.W., Jr. 1996. The pharmacology of mechanogated membrane ion channels. Pharmacol. Rev. 48:231-252.

35. Thastrup, O., et al. 1989. Thapsigargin, a novel molecular probe for studying intracellular calcium release and storage. Agents Actions. 27:17-23.

36. Markram, H., and Segal, M. 1991. Calcimycin potentiates responses of rat hippocampal neurons to N-methyl-D-aspartate. Brain Res. 540:322-324.

37. Garrido, A., Garrido, F., Guerra, R., and Valenzuela, A. 1989. Ingestion of high doses of fish oil increases the susceptibility of cellular membranes to the induction of oxidative stress. Lipids. 24:833-835.

38. D'Aquino, M., et al. 1991. Effect of fish oil and coconut oil on antioxidant defence system and lipid peroxidation in rat liver. Free Radic. Res. Commun. 12-13:147-152.

39. Graf, E., Mahoney, J.R., Bryant, R.G., and Eaton, J.W. 1984. Iron-catalyzed hydroxyl radical formation. Stringent requirement for free iron coordination site. J. Biol. Chem. 259:3620-3624.

40. Hnatowich, D.J., et al. 1983. Radioactive labeling of antibody: a simple and efficient method. Science. 220:613-615

41. Fisher, E.A., et al. 1997. The degradation of apolipoprotein B100 is mediated by the ubiquitinproteasome pathway and involves heat shock protein 70. J. Biol. Chem. 272:20427-20434.

42. Mitchell, D.M., et al. 1998. Apoprotein B100 has a prolonged interaction with the translocon during which its lipidation and translocation change from dependence on the microsomal triglyceride transfer protein to independence. Proc. Natl. Acad. Sci. U. S. A. 95:14733-14738.

43. Rensen, P.C., et al. 2000. Apolipoprotein E is resistant to intracellular degradation in vitro and in vivo. Evidence for retroendocytosis. J. Biol. Chem. 275:8564-8571.
44. Kris-Etherton, P.M., and Yu, S. 1997. Individual fatty acid effects on plasma lipids and lipoproteins: human studies. Am. J. Clin. Nutr. 65(Suppl. 1):1628S-1644S

45. Kendrick, J.S., and Higgins, J.A. 1999. Dietary fish oils inhibit early events in the assembly of very low density lipoproteins and target ApoB for degradation within the rough endoplasmic reticulum of hamster hepatocytes. J. Lipid Res. 40:504-514.

46. Lin, Y., et al. 1995. Differential effects of eicosapentaenoic acid on glycerolipid and apolipoprotein B metabolism in primary human hepatocytes compared to HepG2 cells and primary rat hepatocytes. Biochim. Biophys. Acta. 1256:88-96.

47. Wang, H., Yao, Z., and Fisher, E.A. 1994. The effects of n-3 fatty acids on the secretion of carboxyl-terminally truncated forms of human Apoprotein B. J. Biol. Chem. 269:18514-18520.

48. Borchardt, R.A., and Davis, R.A. 1987. Intrahepatic assembly of very low density lipoproteins. Rate of transport out of the endoplasmic reticulum determines rate of secretion. J. Biol. Chem. 262:16394-16402.

49. Linden, D., et al. 2002. Influence of peroxisome proliferator-activated receptor alpha agonists on the intracellular turnover and secretion of apolipoprotein (Apo) B-100 and ApoB-48. J. Biol. Chem. 277:23044-23053.

50. Wang, H., Chen, X., and Fisher, E.A. 1993. N-3 fatty acids stimulate intracellular degradation of Apoprotein B in rat hepatocytes. J. Clin. Invest. 91:1380-1389.

51. Aalto-Setala, K., et al. 1992. Mechanism of hypertriglyceridemia in human apolipoprotein (Apo) CIII transgenic mice. Diminished very low density lipoprotein fractional catabolic rate associated with increased Apo CIII and reduced Apo E on the particles. J. Clin. Invest. 90:1889-1900.

52. Siri, P., et al. 2001. Post-transcriptional stimulation of the assembly and secretion of triglyceride-rich apolipoprotein B lipoproteins in a mouse with selective deficiency of brown adipose tissue, obesity, and insulin resistance. J. Biol. Chem. 276:46064-46072.

53. Harris, W.S. 1989. Fish oils and plasma lipid and lipoprotein metabolism in humans: a critical review. J. Lipid Res. 30:785-807.

54. Chen, K., Vita, J.A., Berk, B.C., and Keaney, J.F., Jr. 2001. c-Jun N-terminal kinase activation by hydrogen peroxide in endothelial cells involves SRC-dependent epidermal growth factor receptor transactivation. J. Biol. Chem. 276:16045-16050.

55. Mahadev, K., et al. 2001. Hydrogen peroxide generated during cellular insulin stimulation is integral to activation of the distal insulin signaling cascade in 3T3-L1 adipocytes. J. Biol. Chem. 276:48662-48669.

56. Grune, T., Reinheckel, T., and Davies, K.J. 1997. Degradation of oxidized proteins in mammalian cells. FASEB J. 11:526-534.

57. Rashid, K.A., Hevi, S., Chen, Y., Le Caherec, F., and Chuck, S.L. 2002. A proteomic approach identifies proteins in hepatocytes that bind nascent apolipoprotein B. J. Biol. Chem. 277:22010-22017.

58. Fazio, S., and Yao, Z. 1995. The enhanced association of apolipoprotein $\mathrm{E}$ with apolipoprotein B-containing lipoproteins in serum-stimulated hepatocytes occurs intracellularly. Arterioscler. Thromb. Vasc. Biol. 15:593-600.

59. Miyata, M., and Smith, J.D. 1996. Apolipoprotein E allele-specific antioxidant activity and effects on cytotoxicity by oxidative insults and beta-amyloid peptides. Nat. Genet. 14:55-61.

60. Curtiss, L.K., and Boisvert, W.A. 2000. Apolipoprotein E and atherosclerosis. Curr. Opin. Lipidol. 11:243-251.

61. Maugeais, C., Tietge, U.J., Tsukamoto, K., Glick, J.M., and Rader, D.J. 2000. Hepatic apolipoprotein E expression promotes very low density lipoprotein-apolipoprotein B production in vivo in mice. J. Lipid Res. 41:1673-1679. 
62. Fong, L.G., Parthasarathy, S., Witztum, J.L., and Steinberg, D. 1987. Nonenzymatic oxidative cleavage of peptide bonds in Apoprotein B-100. J. Lipid Res. 28:1466-1477.

63. Edelstein, C., Nakajima, K., Pfaffinger, D., and Scanu, A.M. 2001. Oxidative events cause degradation of ApoB-100 but not of Apo[a] and facilitate enzymatic cleavage of both proteins. J. Lipid Res. 42:1664-1670.

64. Leray, C., Wiesel, M.L., Freund, M., Cazenave, J.P., and Gachet, C. 2001. Long-chain n-3 fatty acids specifically affect rat coagulation factors dependent on vitamin $\mathrm{K}$ : relation to peroxidative stress. Arterioscler. Thromb. Vasc. Biol. 21:459-465.

65. Song, J.H., Fujimoto, K., and Miyazawa, T. 2000. Polyunsaturated (n-3) fatty acids susceptible to peroxidation are increased in plasma and tissue lipids of rats fed docosahexaenoic acid-containing oils. J. Nutr. 130:3028-3033.

66. Kubo, K., Saito, M., Tadokoro, T., and Maekawa, A. 2000. Preferential incorporation of docosahexaenoic acid into nonphosphorus lipids and phosphatidylethanolamine protects rats from dietary DHA-stimulated lipid peroxidation. J. Nutr. 130:1749-1759.

67. Fremont, L., Gozzelino, M.T., Franchi, M.P., and Linard, A. 1998. Dietary flavonoids reduce lipid peroxidation in rats fed polyunsaturated or monounsaturated fat diets. J. Nutr. 128:1495-1502.

68. Takahashi, M., et al. 2002. Fish oil feeding alters liver gene expressions to defend against PPARalpha activation and ROS production. Am. J. Physiol. Gastrointest. Liver Physiol. 282:G338-G348.
69. Meydani, M., et al. 1991. Effect of long-term fish oil supplementation on vitamin E status and lipid peroxidation in women. J. Nutr. 121:484-491.

70. Allard, J.P., Kurian, R., Aghdassi, E., Muggli, R., and Royall, D. 1997. Lipid peroxidation during n-3 fatty acid and vitamin E supplementation in humans. Lipids. 32:535-541.

71. Foulon, T., et al. 1999. Effects of fish oil fatty acids on plasma lipids and lipoproteins and oxidantantioxidant imbalance in healthy subjects. Scand. J. Clin. Lab. Invest. 59:239-248.

72. Shepherd, J., et al. 1980. Effects of saturated and polyunsaturated fat diets on the chemical composition and metabolism of low density lipoproteins in man. J. Lipid Res. 21:91-99.

73. Turner, J.D., Le, N.A., and Brown, W.V. 1981. Effect of changing dietary fat saturation on lowdensity lipoprotein metabolism in man. Am. J. Physiol. 241:E57-E63.

74. Cortese, C., et al. 1983. Modes of action of lipidlowering diets in man: studies of apolipoprotein B kinetics in relation to fat consumption and dietary fatty acid composition. Eur. J. Clin. Invest. 13:79-85.

75. Spady, D.K. 1992. Hepatic clearance of plasma low density lipoproteins. Semin. Liver Dis. 12:373-385.

76. Castellani, L.W., et al. 1998. Mapping a gene for combined hyperlipidaemia in a mutant mouse strain. Nat. Genet. 18:374-377.

77. Bodnar, J.S., et al. 2002. Positional cloning of the combined hyperlipidemia gene Hyplip1. Nat. Genet. 30:110-116.

78. Jiang, X.C., et al. 2002. Phospholipid transfer protein (PLTP) deficiency in hepatocytes reduces the secretion of Apoprotein B (ApoB) through a proteolytic pathway that is stimulated by intracellular oxidants and PI3 kinases and inhibited by vitamin E. Circulation. 106(Suppl.):7. (Abstr.)

79. Brown, B.G., Cheung, M.C., Lee, A.C., Zhao, X.Q., and Chait, A. 2002. Antioxidant vitamins and lipid therapy: end of a long romance? Arterioscler. Thromb. Vasc. Biol. 22:1535-1546.

80. Williams, K.J., Scalia, R., Mazany, K.D., Rodrigueza, W.V., and Lefer, A.M. 2000. Rapid restoration of normal endothelial functions in genetically hyperlipidemic mice by a synthetic mediator of reverse lipid transport. Arterioscler. Thromb. Vasc. Biol. 20:1033-1039.

81. Levine, D.M., and Williams, K.J. 1997. Automated measurement of mouse apolipoprotein B: convenient screening tool for mouse models of atherosclerosis. Clin. Chem. 43:669-674.

82. Sparks, C.E., et al. 1986. Insulin effects on apolipoprotein B lipoprotein synthesis and secretion by primary cultures of rat hepatocytes. Metabolism. 35:1128-1136.

83. Stillemark, P., et al. 2000. The assembly and secretion of apolipoprotein B-48-containing very low density lipoproteins in McA-RH7777 cells. J. Biol. Chem. 275:10506-10513.

84. Niehaus, W.G.J., and Samuelsson, B. 1968. Formation of malonaldehyde from phospholipid arachidonate during microsomal lipid peroxidation. Eur. J. Biochem. 6:126-130.

85. Ohkawa, H., Ohishi, N., and Yagi, K. 1979. Assay for lipid peroxides in animal tissues by thiobarbituric acid reaction. Anal. Biochem. 95:351-358. 